\title{
The next generation of biopanning: next gen sequencing improves analysis of bacterial display libraries
}

\author{
Sarah D. Stellwagen ${ }^{1,2}$, Deborah A. Sarkes ${ }^{1 *}$, Bryn L. Adams ${ }^{1}$, Mia A. Hunt ${ }^{1,3}$, \\ Rebecca L. Renberg ${ }^{1,3}$, Margaret M. Hurley ${ }^{1}$ and Dimitra N. Stratis-Cullum ${ }^{1}$
}

\begin{abstract}
Background: Bacterial surface display libraries are a popular tool for novel ligand discovery due to their ease of manipulation and rapid growth rates. These libraries typically express a scaffold protein embedded within the outer membrane with a short, surface-exposed peptide that is either terminal or is incorporated into an outer loop, and can therefore interact with and bind to substrates of interest.

Results: In this study, we employed a novel bacterial peptide display library which incorporates short 15-mer peptides on the surface of E. coli, co-expressed with the inducible red fluorescent protein DsRed in the cytosol, to investigate population diversity over two rounds of biopanning. The naive library was used in panning trials to select for binding affinity against 3D printing plastic coupons made from polylactic acid (PLA). Resulting libraries were then deep-sequenced using next generation sequencing (NGS) to investigate selection and diversity.

Conclusions: We demonstrated enrichment for PLA binding versus a sapphire control surface, analyzed population composition, and compared sorting rounds using a binding assay and fluorescence microscopy. The capability to produce and describe display libraries through NGS across rounds of selection allows a deeper understanding of population dynamics that can be better directed towards peptide discovery.
\end{abstract}

Keywords: Bacterial display, Directed evolution, Peptide display, Biopanning, NGS

\section{Background}

Natural selection has resulted in elegant solutions to the pressures that organisms face. On a macro scale, examples include the cryptic shape and coloration of stick insects, or the specialized feeding organs of mosquitoes, and microscopically, ligand binding and enzymes. Artificially harnessing the power of natural selection has not only provided useful organisms at a larger scale that have shaped culture and diet (e.g. agricultural varieties of plants and animals), but has also contributed to discovering molecular tools that have influenced disciplines from medicine to materials research. Selection of particular molecular interactions is key to natural and engineered antibody-antigen recognition for diagnostics, new

*Correspondence: deborah.a.sarkes.civ@mail.mil

'Biotechnology Branch, CCDC US Army Research Laboratory, 2800 Powder Mill Rd, Adelphi, MD, 20783 USA

Full list of author information is available at the end of the article materials, and an understanding of chemical interactions [1-4]. Peptide discovery that exploits artificial selection pressures to shape diverse peptide libraries have led to a wide variety of unique sequences for targeted use [5-9]. Understanding library diversity, how peptide populations evolve during the discovery process, as well as methods to analyze diversification on a deeper scale are important for improving discovery techniques that rapidly produce unique molecules for purpose-driven benefit.

Surface display libraries employ polypeptides (including peptides, scaffold proteins, and antibody fragments) typically presented at the surface of phage coats or bacterial, yeast, or mammalian cell membranes. Each organism expresses a unique, random sequence with the potential to interact with specific antigenic or molecular targets [10]. After incubating a library with a substrate of interest, the unbound members are washed away while the remaining bound members are amplified and used for further 
screening under more stringent conditions or analyzed to understand binding interactions. While phage display has been the traditional system used for ligand screening, bacterial display libraries provide advantages due to ease of manipulation, as phage reinfection steps are eliminated, and fast growth rates, which are important factors for rapid discovery of binding peptides to assess new and emergent threats $[6,8,9]$.

Historically, peptide libraries were assumed to be highly diverse, unbiased sequence compilations ready for selection against a target material, and examination of the enriched peptides was limited by Sanger sequencing of a few hundred sequences. High throughput next generation sequencing (NGS) technologies now provide an avenue to investigate populations at a much broader scale [11-13]. We use a newly generated library (Fig. 1) to assess diversity and follow population evolution across rounds of biopanning against polylactic acid (PLA) 3D printing plastic. This library relies on a previously engineered outer membrane scaffold protein (eCPX) that co-presents random 15-mer peptides for screening on the $\mathrm{N}$-terminus, and a fixed $\mathrm{P} 2 \mathrm{X}$ peptide at the $\mathrm{C}$-terminus to monitor expression via binding to the fluorescent protein YPet-Mona [14-16]. If a stop codon were present in the random peptide, P2X binding by YPet-Mona (Fig. $1 \mathrm{~b}$ and $\mathrm{c}$ ) would not occur because the remainder of the eCPX scaffold would not be produced or shuttled to the outer membrane. This interaction can be monitored using Fluorescence-Activated Cell Sorting (FACS) [17]. Additionally, we incorporated inducible DsRed fluorescence into the vector cassette (Fig. 1) allowing visualization to compare binding efficiency as an alternative method to indirect binding assays [6]. Indirect binding assays measure the cell proliferation of bound cells after a recovery stage to extrapolate and relatively quantify the number of bound library members. Serial dilution and plating of the resulting culture of amplified cells allows counting of colony forming units, while visualizing and quantifying cells directly bound to nonconductive materials by scanning electron microscopy (SEM) would require sputter coating with a metal such as gold. Here, we analyzed peptide sequences from the naive library and after each round of biopanning using Illumina NGS technology (Fig. 2) to understand the overall dynamics of population selection, and then visualized the bound cells directly on the PLA surface using the incorporated DsRed protein with fluorescence microscopy. The biopanning process is described in detail in Fig. 3, and is similar to previously described methods for isolating aluminum binders using a related eCPX-based library [6], except that here we additionally co-expressed the DsRed protein for direct visualization.

\section{Results}

\section{Analysis of library diversity}

We used Illumina next generation sequencing technology to assess peptide diversity for a newly created bacterial display library and after 2 rounds of biopanning against PLA 3D printing plastic (Figs. 2 and 3). The PLA
A)

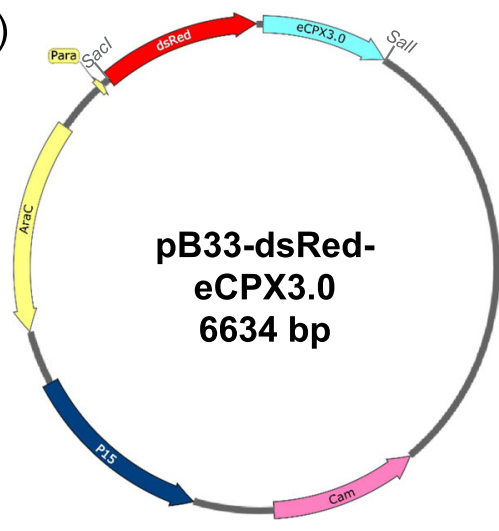

B)

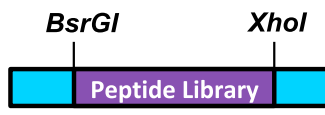

C)

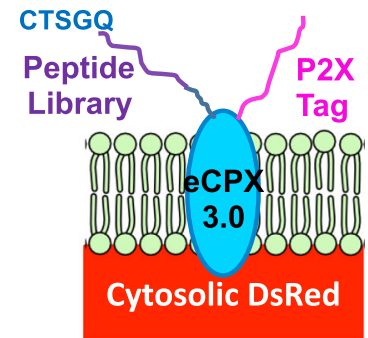

Fig. 1 pB33-dsRed-eCPX3.0 Peptide Library Design. a Plasmid map of DsRed-peptide display expression plasmid showing relative location and orientation of genes. b Diagram of eCPX3.0 gene from 5' to $3^{\prime}$ indicating location of 15-mer peptide display library sequence, restriction sites flanking the library, and the location of the C-terminal P2X tag. c Schematic of mature eCPX3.0 display scaffold embedded in the cell membrane with N-terminal peptide library (preceded by CTSGQ) and C-terminal P2X tag displayed at the cell surface, and DsRed accumulating in the cytosol for cell visualization. The N-terminal peptide library contains random sequences, while the fixed C-terminal P2X tag allows expression monitoring through binding to the fluorescent protein YPet-Mona 


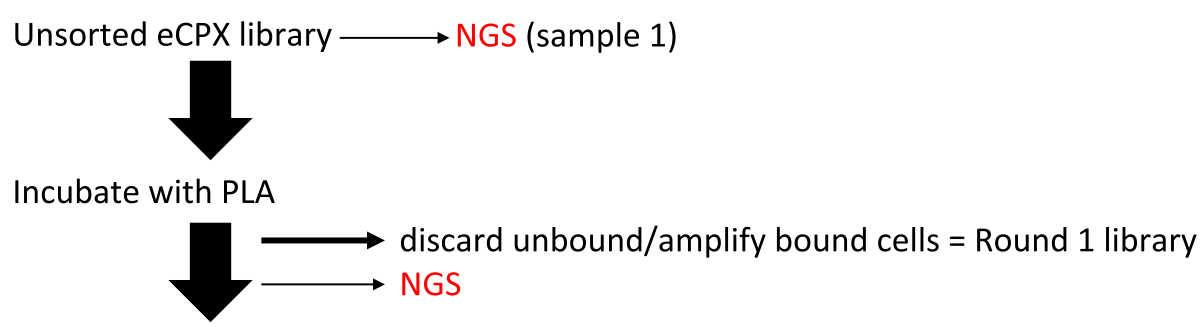

Incubate with PLA

$\longrightarrow$ discard unbound/amplify bound cells = Round 2 library

Fig. 2 Flow Chart for Biopanning and NGS Sequencing. The naive, unsorted library was both sequenced and used for biopanning against PLA 3D printing plastic. After washing the PLA sample and amplifying bound library members, the Round 1 library was sequenced and again panned against fresh PLA. The final, Round 2 library was sequenced along with the naive and Round 1 libraries

coupons were prepared by melting filaments together as described in Fig. 4 and the experimental methods. Illumina NGS sequencing resulted in 1,212,302 reads from the naive library, 1,418,018 reads from the Round 1 library, and 1,405,367 reads from the Round 2 library (Additional file 1: Table S1). The strategy for filtering sequences from this data is outlined in Fig. 5, showing examples of valid, useable sequence, and invalid sequences containing stop codons, frame shifts, or no peptide insert. We define 'unique' sequences as the total complement of different amino acid sequences for any category. For example, a valid insert with more than one representative is counted as a single representative of the 'unique' category for valid sequences. Valid inserts (inserts in the

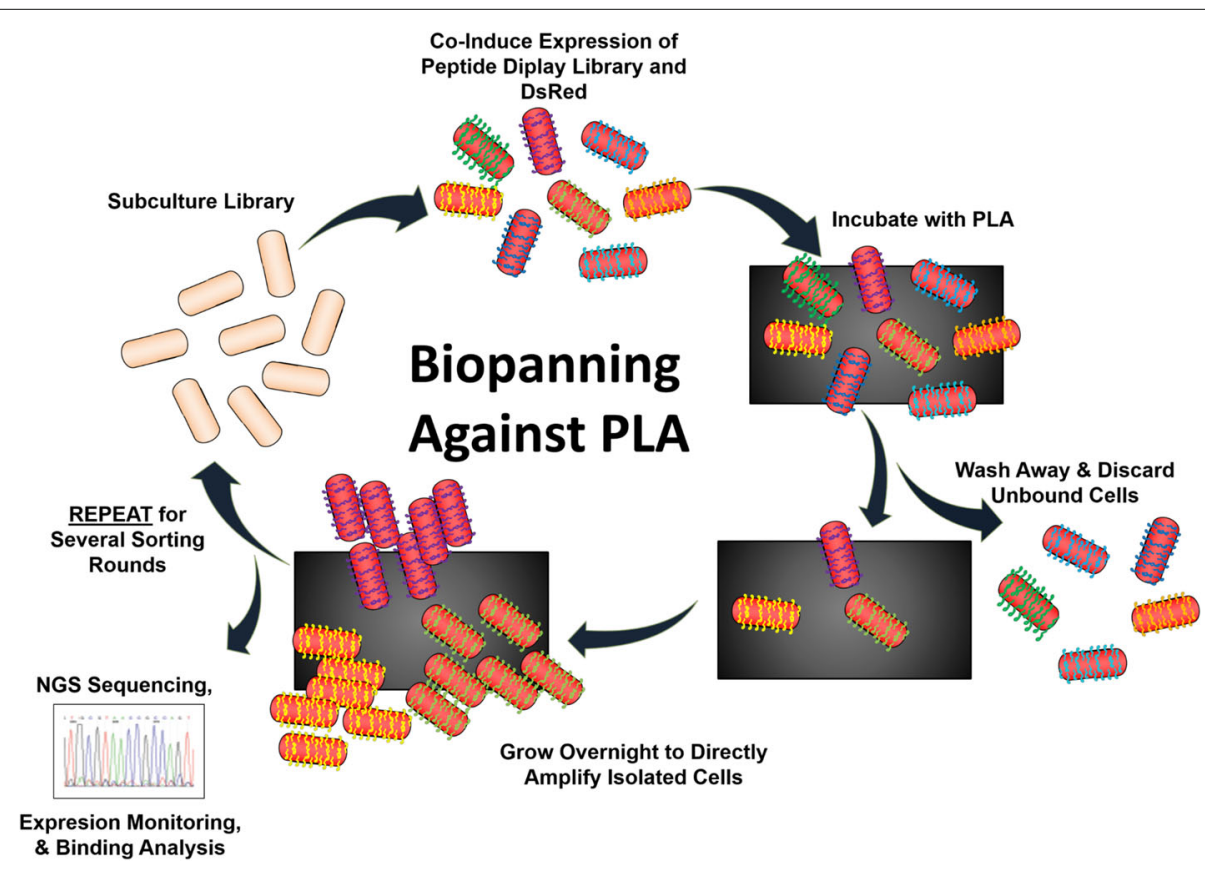

Fig. 3 Biopanning experimental design schematic. Biopanning is a cyclical process that begins with subculturing the naive, unsorted library. Co-expression of cytosolic DsRed and membrane-localized peptides from the pB33-dsRed-eCPX3.0 library is achieved by induction with L-arabinose during log phase of cell growth. The induced library is then incubated with PLA 3D printing plastic for 15 min with shaking. Next, the PLA coupons were washed vigorously with PBS $1 \%$ Tween for 30 min to remove unbound cells, and then placed in LB +Cm with 2\% D-Glucose media to proliferate and recover the bound library. The presence of glucose halts the production of new copies of membrane-displayed peptides, which are slowly diluted during cell proliferation, along with the cytosolic DsRed. The process is repeated for sorting rounds 1 and 2, changing only the starting library material (using the previous sorting round). After each round, NGS sequencing was performed and peptide expression level monitored. Bound cells can be visualized directly on the PLA using fluorescence microscopy due to the presence of DsRed, and binding level can also be indirectly compared using regrowth assays and cell counting 


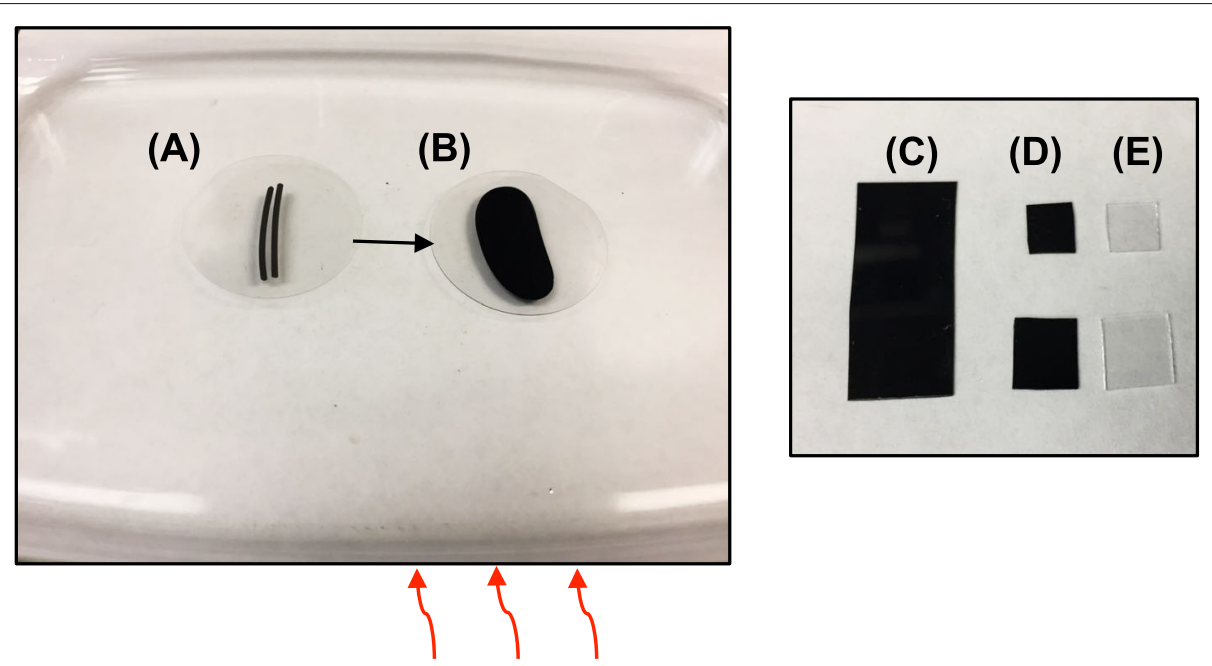

Fig. 4 Polylactic Acid (PLA) and sapphire material sample preparation. Two pieces cut from a spool of PLA printing filament $\sim 4 \mathrm{~cm}$ in length were placed on a Pyrex baking dish under a sapphire wafer (a) and then heated with a hot plate until melted (b). After melting, PLA pieces were removed from the Pyrex dish and sapphire wafers, then cut into coupons for biopanning trials (c), or cut into smaller pieces used for binding quantification (d) with equally sized sapphire pieces (e)

correct reading frame without stop codons) comprised $38 \%$ of all sequences in the naive library, $85 \%$ of which were unique (or $33 \%$ of the total library; Fig. 6). After one round of selection, valid inserts increased to represent $42 \%$ of the library population; however, $11 \%$ of these ( $5 \%$ of the total library) were unique. The second round of sorting resulted in more than half the library's representation as valid inserts (55\%), with an even further decrease in unique valid inserts ( $3 \%$ of valid inserts were unique or $2 \%$ of the total library). Library members with inserts that resulted in a frame shift, contained stop codons, or that had an empty scaffold made up $27 \%, 21 \%$, and $13 \%$ of the naive library population, respectively. A general reduction was observed for members from these categories across panning rounds, accompanied by the distinct reduction in the representation of unique individuals, as compared to valid sequences (Fig. 6).

The most frequent sequences represented in the naive, unsorted library generally consisted of single-residue inserts (Table 1), although the bulk of the unsorted library consisted of 15 -mers $(375,287$ out of 396,641 unique sequences, or 95\%). Leucine alone had the highest representation and was identified 32 times $(0.003 \%)$, while the most frequent 15-mer (PRKTLKGTLTVPSYI) was identified 8 times $(0.0007 \%)$. The most common insert sequence discovered in the Round 1 library, a 15-mer (VLPQTSFFAATCRRS), was represented 115 times (0.01\%), which increased to 283 in Round 2. 15-mers were again the main component of the library in Round 1, consisting of 64,507 out of 67,885 sequences (95\%). The most common insert sequence discovered in Round 2, also a 15-mer (CHISPEKRRLIVCAD), was represented 4,619 times (0.38\%), which increased from 28 in Round 1. In Round 2, 15 -mers comprised 20,903 of 21,931 unique sequences (95\%). The frequency of the population's top sequence increased 4-fold from the naive library to Round 1, and 40-fold from Round 1 to Round 2.

Representation of all 20 amino acids as a decimal of the overall (i.e. all unique sequences and not just 15-mer) library character was generated round by round, both including and excluding frequency of sequence occurrence (Additional file 1: Figure S1). This represents a

\begin{tabular}{|c|c|}
\hline & xxxxxx_left_flanking_sequence___insert___right_flanking_sequence_xxxxx \\
\hline A) & 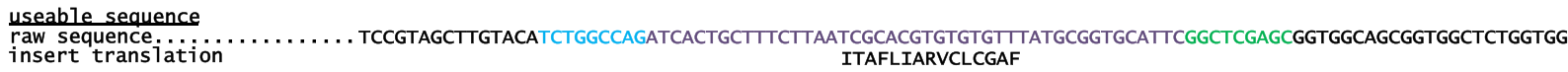 \\
\hline B) & $\begin{array}{l}\text { stop codons } \\
\text { raw sequence } \\
\text { insert trans } i a \operatorname{ion}\end{array}$ \\
\hline C) & $\begin{array}{l}\text { frame shift } \\
\text { raw sequence ................ TCCGTAGCTTGTACATCTGGCCAGAATCGGTTATCCATTCTTCATGCTTATCCCGCAACTGTATCACGGGCTCGAGCGGTGGCAGCGGTGGCTCTGGTGGT } \\
\text { insert base \# }\end{array}$ \\
\hline D) & $\begin{array}{l}\text { no insert } \\
\text { raw sequence................ TCCGTAGCTTGTACATCTGGCCAGGGCTCGAGCGGTGGCAGCGGTGGCTCTGGTGG }\end{array}$ \\
\hline & $\begin{array}{l}\text {. } 5 \text { Strategy for filtering NGS sequences. A valid insert sequence consists of correct flanking regions, an insert within the correct reading frame } \\
\text { ically } 45 \text { bp), and without stop codons (a). Sequences that translate into an insert with stop codons (b), result in a frame shift (c), or that have no } \\
\text { art (d) are filtered into separate categories }\end{array}$ \\
\hline
\end{tabular}




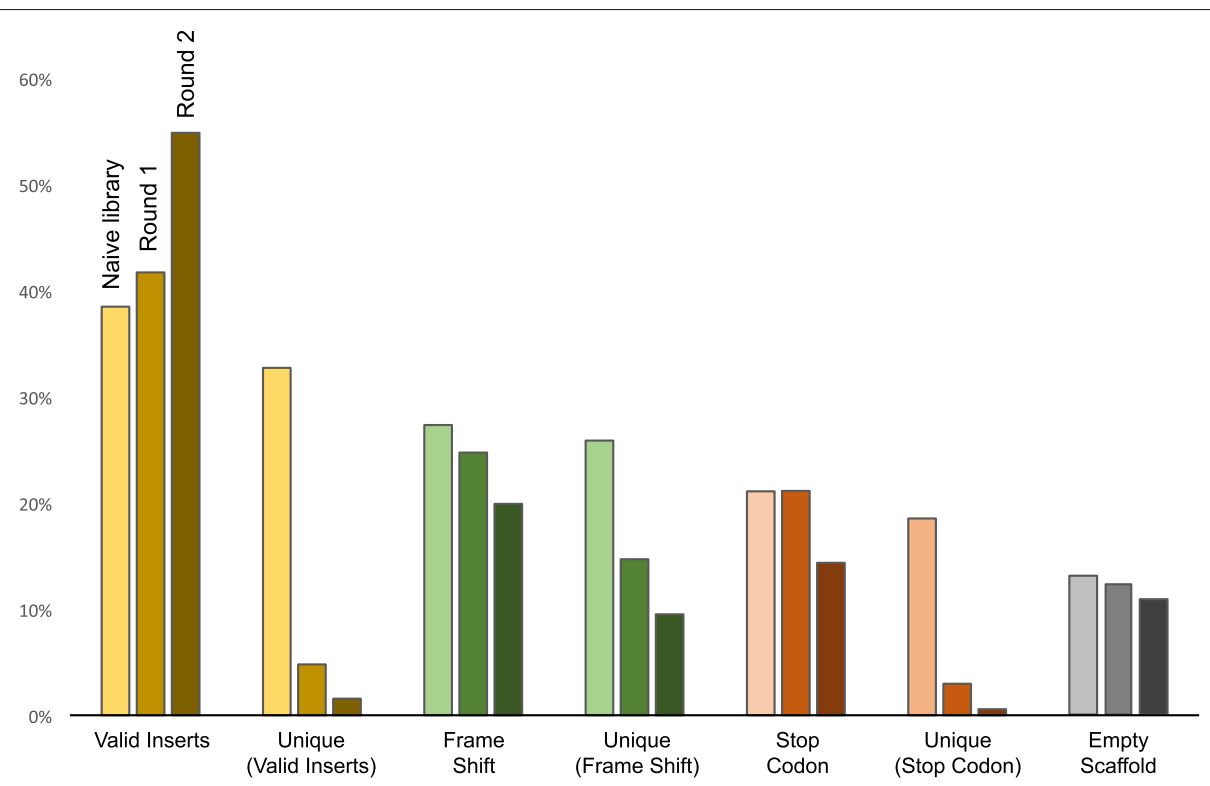

Fig. 6 Percent representation of each insert type from the naive and sorted libraries after NGS sequencing. Colors are grouped by total representation of the library followed by frequency of each unique insert for each category, and are in the order Naive library, Round 1, and Round 2. The Empty Scaffold category does not have a frequency representation, as members cannot be tracked across panning rounds. Raw sequence counts can be found in Additional file 1: Table S1

flat numeric analysis of current sequencing data with no correction for background probabilities from previous genomic analyses. Despite that, this information is useful as a depiction of both static and dynamic characteristics of the library. There is a consistently high representation of hydroxyl containing residues $\mathrm{S}$, $\mathrm{T}$, basic residue $\mathrm{R}$, and hydrophobic residue L. Additionally there is underrepresentation from acidic residues D and $\mathrm{E}$ and hydrophilic residues $\mathrm{N}$ and $\mathrm{Q}$. Round by round modifications to the distribution are subtle, but there is an increase in hydrophilic residues $\mathrm{C}$ and $\mathrm{Y}$, an increase in hydrophobic residues $M$ and $L$, an increase in aromatic residues $F$ and $\mathrm{W}$, and a decrease in $\mathrm{D}, \mathrm{E}, \mathrm{N}$ and $\mathrm{Q}$. These trends are visible regardless of the incorporation of sequence frequency.

Analysis of sequence motifs in round by round 15-mer sequence character by the pLogo generator [18] further examines the inclusion of background probability statistics for E. coli $\mathrm{K} 12$ and provides an assessment of spatial organization within the sequence (Additional file 1: Figure S2). The trends seen from the rough numbers in Additional file 1: Figure S1 are accentuated in this representation. There is an up-regulation of hydrophilic residues $S, T, C, Y$, up-regulation of aromatic residue $\mathrm{W}$ and basic residue $\mathrm{R}$, down-regulation of acidic residues $\mathrm{D}$ and $\mathrm{E}$, and down-regulation of nonpolar residues $\mathrm{A}, \mathrm{G}$ and $\mathrm{L}$. The increase in $\mathrm{M}, \mathrm{C}$, and $\mathrm{W}$ by round is more visible in this representation, as is the down-regulation by round of $\mathrm{E}$ and $\mathrm{A}$. By round 2, dominance of key residues $\mathrm{C}, \mathrm{W}, \mathrm{S}, \mathrm{M}$ and $\mathrm{R}$ are clearly visible. There is very little spatial dependence on this up- (or down-) regulation, as befits interactions with a surface and with sequences which are largely expected to be loose coil in structure (as can be seen from the list in Table 1). There are further differences in sequence character in round 2 when comparing the pLogo generated motif of the entire 15-mer population of the library (Additional file 1: Figure S3).

The increase in cysteine after sorting is particularly interesting since it could have both positive and negative effects on the peptide library. Cysteine could help constrain any structural component of the peptide that aids in binding affinity or specificity by creating disulfide bonds that increase rigidity [19]. However, when several cysteine residues are present in the library, unwanted intermolecular disulfide bonding could also occur, which could cause clumping of the displayed cells and interfere with isolation of individual peptides within the population for characterization. The peptides fused by disulfide bonds could have cooperative binding effects, which is not a problem in and of itself, but could be difficult to understand and replicate off-cell. In this study it was shown that the percentage of peptide sequences with zero cysteines decreases round by round, and the percentage of sequences containing more than a single cysteine changes only slightly (Additional file 1 : Table S2). Since the increase in cysteine is primarily due to an increase in peptides containing a single cysteine, intra-sequence disulfide binds are not significantly changing and probably don't have much effect on peptide rigidity. 
Table 1 Top 40 most frequent sequences from the naive library and after 2 rounds of biopanning

\begin{tabular}{|c|c|c|c|c|c|c|}
\hline & Naive Library & No. Seqs & Round 1 & No. Seqs & Round 2 & No. Seqs \\
\hline 1 & L & 32 & VLPQTSFFAATCRRS & 115 & CHISPEKRRLIVCAD & 4619 \\
\hline 2 & $P$ & 31 & SNRLRGENLTFRRHK & 110 & YPLCSTYGNVLVGGS & 3989 \\
\hline 3 & $\mathrm{R}$ & 30 & DRRRKIERYDAQISV & 89 & PLKQGIYETTSTKGT & 3321 \\
\hline 4 & S & 22 & $\mathrm{~T}$ & 87 & YGSRTFPVCSTVHRR & 2185 \\
\hline 5 & A & 19 & S & 84 & YRLMEMEEYHKDVFA & 1985 \\
\hline 6 & $\mathrm{~T}$ & 13 & PAKGSIKNPILLGIR & 74 & KHLLCVMQGGTAFRG & 1783 \\
\hline 7 & E & 12 & QPLSESLEDKTSIDV & 74 & RIFGFLSCKPNIIKL & 1094 \\
\hline 8 & $\mathrm{H}$ & 11 & SS & 73 & IVNRMWCLISVISQA & 921 \\
\hline 9 & SP & 11 & THNARRLMPCIDASD & 73 & FWGLYPLFCCTGCSP & 909 \\
\hline 10 & Y & 11 & YPGRMEALFAHVSNA & 73 & SKLFSSWVLCACWHC & 778 \\
\hline 11 & Q & 10 & RDPSWRAETVNCGNL & 69 & AVLAIYPNSLYWLQA & 656 \\
\hline 12 & $\pi$ & 10 & RHQNPQEEI & 69 & YIAMILHGFSQAFPG & 650 \\
\hline 13 & V & 10 & YTYHGRNSERLLRMK & 69 & LLFSFFNCFTGNDGQ & 636 \\
\hline 14 & F & 9 & TQDETRRGGMSTTSS & 68 & NKWSPRTDFNTESVR & 623 \\
\hline 15 & GPCLETCLTA & 9 & HTYCVHPSMATCWHY & 66 & HAPWECLISVLASAH & 616 \\
\hline 16 & ERSVG & 8 & ANLPDSHHIAHNKDT & 65 & ARGLLTAISFYAFQC & 602 \\
\hline 17 & LT & 8 & AMPDFVILDHGVTRR & 64 & RLWMRSPTSTAGGIR & 601 \\
\hline 18 & PRKTLKGTLTVPSYI & 8 & KNSLTCCYG & 63 & AERLHQITVMLLRSF & 595 \\
\hline 19 & SG & 8 & RRNYIHIGAKAAGRS & 63 & LSNLLHYLYALGQPG & 595 \\
\hline 20 & SKAPLRKTNMKLR & 8 & DVLAKYSSLERQDLP & 62 & PILLCYVLYCEYASS & 587 \\
\hline 21 & TS & 8 & SVAATCWRYWYDKPL & 62 & SWTMLICQLHSYLYT & 583 \\
\hline 22 & ATYYLQPYVPISEET & 7 & RPPCEEMSINCPGVT & 61 & PRALWEASMWTLAC & 581 \\
\hline 23 & DQLGVL & 7 & TYPCIYA & 61 & AVCKPVGPKAPWRDR & 575 \\
\hline 24 & GPWATWSSYDKWHNR & 7 & VSLIDQCDCTMQSNP & 61 & SSRSLQQNSISRLSM & 571 \\
\hline 25 & GT & 7 & HRRRKPIHLPEYPLP & 60 & IRKQWDFLCLGFLLS & 559 \\
\hline 26 & KS & 7 & RHSICPRFRGLLPHS & 60 & SAFPACGLASYFPWF & 554 \\
\hline 27 & PCNFISKHFVPHRRH & 7 & RML & 60 & GWRHCPAWQKSPWP & 532 \\
\hline 28 & PIVGVYK & 7 & ARTSG & 59 & HSALDVLSWVISSLM & 532 \\
\hline 29 & PR & 7 & DWMKFHVEA & 59 & GLRIQAYALCYWEPV & 526 \\
\hline 30 & SFLL & 7 & GPANWTCAWQAVTAF & 59 & NRAIIGEIAGLIKFL & 522 \\
\hline 31 & TD & 7 & LLVKLGP & 59 & PAAAFFTQVMSLLRQ & 503 \\
\hline 32 & WD & 7 & RGVGTSHPQSVCVKP & 59 & RLHCLERYINLAFLP & 503 \\
\hline 33 & YT & 7 & CIT & 58 & RMHHLMFRDSHSHTD & 502 \\
\hline 34 & AMTSLPSTCRQLHSN & 6 & WLHGVLLNFKKHGVC & 58 & TQKHKTLCFSIRKPG & 494 \\
\hline 35 & AQDRVRLKSFGRASL & 6 & IHSAGRMSINLCVGI & 57 & LRYGIWQVSHIALTS & 492 \\
\hline 36 & CQFNWWANLARIAIG & 6 & IRRTLLGRPVWFFEE & 57 & QSFVMESVLSFGFSF & 492 \\
\hline 37 & EDGPTRLWPLSRAAW & 6 & RSSRSVDPLTYRRNA & 57 & DRRRKIERYDAQISV & 486 \\
\hline 38 & FSLTVFVMDQENVNR & 6 & YQPWREDRRSSAGSA & 57 & PHKLWRLGCFVAMDL & 485 \\
\hline 39 & GDSEGLRMSTLGSSS & 6 & GLVRVFWPTPERGHR & 56 & KTVFCLTVLPRFEAA & 482 \\
\hline 40 & GTIGFLDLPRKTVMR & 6 & AVKAYSTFRQSPTRL & 55 & RHLVSWLCTVLHPDP & 481 \\
\hline
\end{tabular}

\section{Binding assays}

We compared a binding assay using direct visualization that measures fluorescent pixel coverage of microscopy images (Figs. 7a \& 8) with an indirect binding assay that quantifies plated CFUs to estimate library density (Fig. 7b). We incubated PLA and sapphire samples with the naive library, libraries produced after each round of sorting, and negative control cultures. Both assays showed 


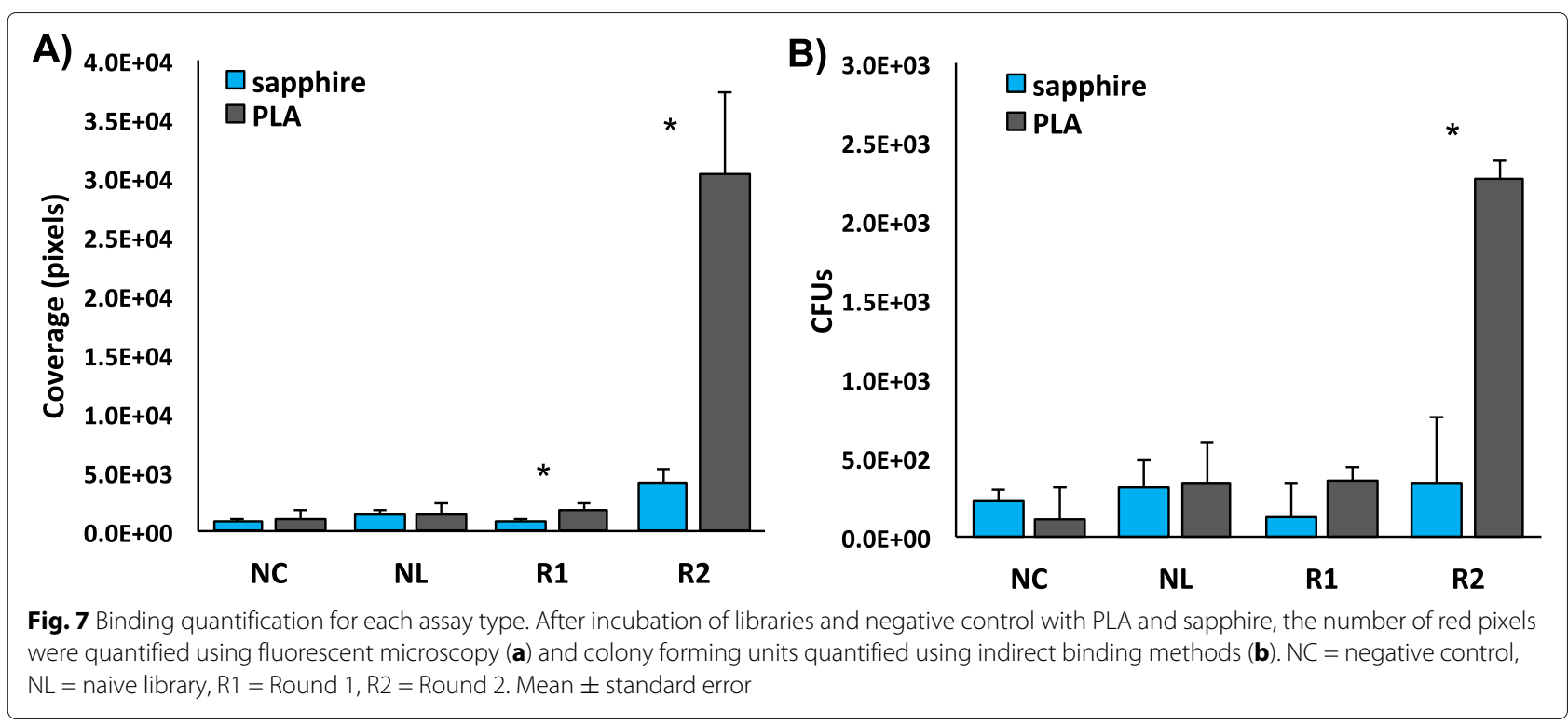

similar trends, and binding was significantly higher on the PLA coupons than sapphire pieces after Round 2 for both visualization $(\mathrm{P}<0.0001)$ and indirect $(\mathrm{P}=0.0015)$ assays, and after Round 1 using the visualization assay $(\mathrm{P}<0.0001)$. There were no differences between the naive library, sorting rounds, and negative control when comparing growth curves (Additional file 1: Figure S4).

Along with the quantified results in Fig. 7, the fluorescence microscopy images shown in Fig. 8 demonstrate that after two rounds of sorting against PLA, cells displaying peptides from the $15 \mathrm{mer}$ library are able to bind the desired material and remain bound after vigorous washing (Fig. 8a). Furthermore, the binding level is higher for PLA (Fig. 8a) as compared to binding to a sapphire wafer (Fig. 8b). The enrichment of binding for PLA over sapphire indicates that the peptides are not just "sticky" in general, but have demonstrated affinity for the material of interest. There is some evidence of cell clumping in Fig. 8a, which may be caused by intermolecular disulfide bonds, discussed above, as a consequence of enrichment for cysteine residues in the library. Additionally, a peptide-free negative control culture, which still displays the empty eCPX scaffold and produces cytosolic DsRed, shows very little binding (Figs. 7 and 8c), indicating that the enriched peptide library is responsible for binding, rather than being caused by either of these over-expressed proteins, or by the cells used to generate the library.

\section{Discussion}

Next generation sequencing technologies allow a deeper understanding of peptide selection from display libraries. For the first time, we used NGS to analyze libraries that employ bacterial display across rounds of biopanning to enrich libraries for surface binding. We sequenced populations from a newly developed, unsorted library $($ naive $=\mathrm{N})$, and after two rounds of selection (Round $1=\mathrm{R} 1$, Round $2=\mathrm{R} 2$ ) against PLA 3D printing plastic (Figs. 5 and 6). Sequencing results confirm population diversity of the
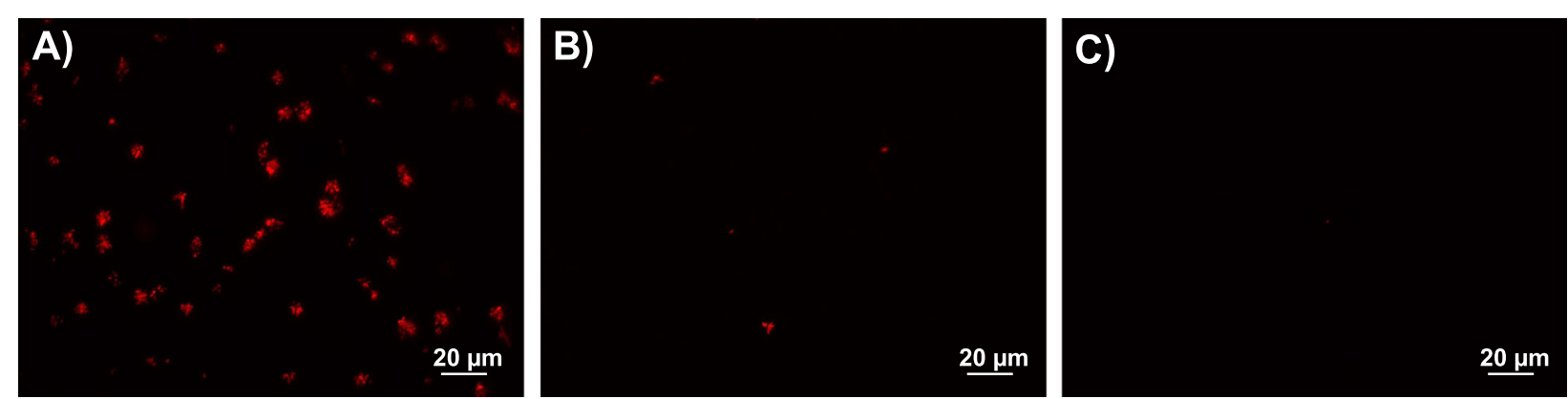

Fig. 8 Fluorescent microscopy images of bound cells. a Round 2 library on PLA, b Round 2 library on sapphire wafer, and c negative control on PLA after 10 min incubation with sample materials and 10 min washing 
naive library and a reduction in this diversity across panning rounds (Fig. 6). Visualization of the Round 2 library demonstrates enrichment of PLA binding peptides after selection (Figs. 7 and 8). Valid, unique inserts represented $33 \%$ of the total naive bacterial display library (Fig. 6), more than double what was observed after deep sequencing of a naive 7-mer phage display library produced using NNK saturation mutagenesis, which recovered only $15 \%$ of sequences with valid, potential binders [20]. The representation of valid inserts increased through successive rounds of biopanning (N: $38 \%, \mathrm{R} 1: 42 \%$, R2: $55 \%$ of the total library); however, the frequency of individual representatives decreases dramatically after just the first round (N: 33\%, R1: 5\%, R2: $2 \%$ of the total library), as adherent members are carried forward while weakly bound members are washed away in increasingly stringent washing steps.

The total fraction of nonbinding library members that display an empty scaffold, or contain sequence that results in a frame shifts or stop codon decreased 3-7\% from the naive library across rounds of sorting (Fig. 6). Interestingly however, the frequency of individual sequences that contain frame shifts or stop codons (we cannot track empty scaffold individuals across rounds as they lack unique insert sequences) decreased more dramatically, reducing the diversity of these sequences $16 \%$ (frame shift) or $18 \%$ (stop codon). It is expected that after applying selective binding pressure, some individuals exhibiting binding not related to peptides or the display scaffold, or that are carried over for other reasons that are less understood (i.e. genomic mutations, growth rate advantages, etc.), would be enriched to some extent due to the natural evolution process. However, unlike the overall population increase observed for individuals that display a valid peptide (Fig. 6), the representation of nonbinding library members does not increase (note binding increase with sorting rounds in Fig. 7), suggesting a stronger association of valid inserts with the PLA surface. Nonbinding library population diversity does exhibit a similar dramatic decrease as compared to valid insert population diversity.

The library used in this study was created to reduce stop codons by employing $15 \mathrm{x}$ NNS saturation mutagenesis, where $\mathrm{N}$ represents any base and $\mathrm{S}$ represents $\mathrm{G}$ or $\mathrm{C}$. This technique produces a library of inserts with a representation of all 20 amino acids, but with an incidence rate of $3 \%$ of the codons in the naive library as stops. With 15-mer insert lengths, this translates to an expected $45 \%$ of sequences containing stop codons if stop codons occur singularly within an insert. Only $24 \%$ percent of our naive library scaffolds with a 15-mer contain at least one stop codon; a further $20 \%$ of these contain at least 2 or more stops. Moreover, a percentage of inserts with frame shifts (27\% of the total library) also must contain stop codons, although it is not possible to distinguish a correct reading frame in order to quantify their frequency. There is a tradeoff between insert length and stop codon occurrence, as longer inserts with more residues have a higher chance of incorporating a stop. For example, libraries with a 7-mer insert length would be expected to have only a $21 \%$ stop codon occurrence. Library generation must balance the benefits of longer inserts that present secondary structure for enhanced binding with maintaining a diverse library of valid inserts free of stop codons.

The amino acid representation within the library does show subtle changes round by round as expected (Additional file 1: Figures S1 and S2). However, we note that the overall shape of the profile stays roughly the same (Additional file 1: Figure S1) and that diversity may be diminished but not extinguished (Fig. 6, Unique Valid Inserts). There is a general increase in residues capable of hydrogen bond donation consistent with the fact that PLA is a chemically generic aliphatic polyester capable of hydrogen bond acceptance through the polymer backbone. We also note an increase in the sulfur containing residues cysteine and methionine. $\mathrm{n} \rightarrow \pi^{*}$ interactions have been noted between cysteine sulfurs and protein backbone carbonyl groups [21] and similar interactions with the PLA backbone may contribute to the up-regulation of $C$ and $M$ clearly shown in the motif representation. Trends shown from the entire library to the top 40 most frequent sequences to the top 10 most frequent sequences in round 2 are also subtle. It is clear that the predominance of $\mathrm{W}$ in the library as a whole is less important in the context of the top 10 PLA binding sequences, while the cysteine interactions mentioned above are clearly in play for all. No true spatial dependence is flagged in any sequence analysis. We note from visual inspection of the top 10 binding sequences in Round 2 that hydrogen bond donors such as R, C, S, Y and T are highly represented.

Assessing populations for binding enrichment on nonantigenic material surfaces using bacterial display libraries in the absence of fluorescence has depended on methods that require additional steps, such as SEM sputter coating for nonconductive surfaces or indirect binding assays $[6,22]$. Indirect binding assays require an additional growth step after material incubation to amplify individual binders, followed by serial dilution and replicated plating of library populations. Our new library incorporates an inducible DsRed-Express2 fluorescent protein to allow direct visualization and quantification of enrichment on sample surfaces to eliminate SEM sample preparation or indirect assays that consume time and laboratory supplies, and avoids potential errors caused by cell growth rate differences. After incubating PLA and sapphire pieces with induced libraries and subsequent washing, binding was quantified by measuring either fluorescent pixel coverage, or the number of CFUs from an indirect assay (Fig. 7). 
Similar trends were observed for each method of quantification, and in addition to significant differences between PLA and sapphire binding from the Round 2 library, the visualization assay also uncovered differences in Round 1 binding not found in the indirect assay (Fig. 7a).

While we demonstrate the enrichment of peptides with affinity for PLA in this work, and that the enriched peptide library is not purely "sticky" since it does not bind to sapphire with similar affinity (Fig. 8b), the primary goal of this work was to demonstrate how NGS and coexpression of fluorescent proteins can be used to enhance data analysis of display libraries in general, rather than to provide an optimized peptide sequence for binding PLA. To further optimize affinity and specificity of peptides for PLA, it could be useful to employ a constrained peptide library, as they have been shown to improve both affinity and specificity [19]. Specificity for PLA, for instance over other plastics, can also be improved by incorporation of negative sorting steps in future studies. Strategies including negative sorting have previously been shown to provide specificity over structurally similar biological targets $[5,17,23]$, and are expected to analogously aid in biopanning peptides towards inorganic materials.

\section{Conclusions}

Bacterial display is a valuable tool for rapidly screening peptide libraries. Our results demonstrate that the library generated for this study is diverse, contains a high proportion of valid inserts compared to phage libraries, and can be used successfully for material surface binding enrichment. Binding sequence motif analysis shows little spatial dependence and a general increase in the representation of hydrogen bond donors to interact with the polyester backbone of PLA. The DsRed-Express2 fluorescent protein provides an effective means for visualizing and quantifying adherent library members as an alternative method to indirect binding assays. Similar co-expression strategies with fluorescent proteins and substrate-specific peptides could also be employed for Förster resonance energy transfer (FRET) or other uses [24]. High-throughput next generation sequencing technology allows researchers to verify initial library diversity, gain a better understanding of the effects of selective pressure applied to library populations, and provide a baseline analysis for improving library design and application.

\section{Methods}

\section{Bacterial strains, plasmids, materials and growth conditions}

All experiments were performed with E. coli MC1061 F' Electrocompetent cells strain SS320 (Lucigen; cat.no. 60512-2) [25]. Bacterial cells were routinely cultured in Luria-Bertani (LB) Miller broth (BD Difco) supplemented with $34 \mu \mathrm{g} / \mathrm{ml}$ chloramphenicol $(\mathrm{LB}+\mathrm{Cm})$. Agar $(15 \mathrm{~g} / \mathrm{L})$ was added for solid media and cultures maintained at $37^{\circ} \mathrm{C}$. Liquid cultures were grown in $14 \mathrm{ml}$ culture tubes (Falcon) and shaken at $250 \mathrm{rpm}$. The eCPX peptide display scaffold was encoded on plasmid pBAD33-eCPX [16] and is available from Addgene. The plasmid was modified by replacing eCPX with eCPX3.0, which includes a P2X peptide displayed on the C-terminus [16]. Additional modification to the Addgene plasmid sequence included mutation of an SfiI site upstream of the peptide insertion site to create a BsrGI site for ease of library insertion. DsRed-Express2 was PCR amplified from commercial vector pCMV-DsRed-Express2 (Takara) and inserted directly upstream of eCPX. The backbone vector was constructed by co-inserting DsRed-Express 2 and the modified eCPX 3.0 sequence into pBAD33-eCPX using SacI and SalI restriction sites to create pB33-dsRed-eCPX3.0 (Fig. 1).

To construct the library, the pB33-dsRed-eCPX3.0 backbone vector was digested with $B s r G I$ and $X h o I$ and purified using DNA Clean and Concentrator kit (Zymo Research). A DNA library of peptide inserts in the form of NNS within the eCPX3.0 gene was commercially synthesized (GeneArt), similarly digested and ligated into the vector backbone using electroligase (New England Biolabs). A total of $1.5 \mu \mathrm{g}$ of newly assembled constructs were then electroporated into $250 \mu$ l electrically competent cells in batches of $1 \mu \mathrm{l}$ DNA with $25 \mu \mathrm{l}$ cells using a $0.1 \mathrm{~cm}$ gap, pre-chilled electroporation cuvette, and pulsed at $1.8 \mathrm{kV}, 10 \mu \mathrm{F}$, and $100 \Omega$. The cuvette was rinsed three times with $1 \mathrm{ml}$ warm SOC and transferred to a 125 $\mathrm{ml}$ vented flask. This process was repeated until the entire volume of pB33-dsRed-eCPX3.0 library was transformed ( $\sim 10$ times). Approximately $70 \mathrm{ml}$ warm SOC was added to the flask and incubated at $37^{\circ} \mathrm{C}$ for $1 \mathrm{~h}$. After recovery in SOC, the library transformants were added to $500 \mathrm{ml}$ of warm $\mathrm{LB}+\mathrm{Cm}$ for $4 \mathrm{~h}$. The culture was then split into five flasks of $1 \mathrm{~L} \mathrm{LB}+\mathrm{Cm}$ and grown overnight. The following day, each liter was pelleted and resuspended in $10 \mathrm{ml} \mathrm{LB}+15 \%$ glycerol. From this, $1 \mathrm{ml}$ frozen library stocks were prepared and stored at $-80^{\circ} \mathrm{C}$, with each stock containing $\sim 10^{11}$ cells.

\section{PLA coupons}

A $1 \mathrm{~kg}$ spool of $1.75 \mathrm{~mm}$ 'True Black' PLA 3D Printer Filament from HATCHBOX@ $囚$ was used to make $1 \times 3 \mathrm{~cm}$ coupons for use in binding trials. PLA plastic filament for 3D printing is delivered in spools, and must be melted into thin pieces between smooth-surfaced materials. Two $\sim 4$ $\mathrm{cm}$ pieces of PLA filament were placed side-by-side on the bottom of a Pyrex baking dish (which maintains a smooth surface and can withstand rapid changes in heat) and set onto a hot plate heated to level $6 / 10$, or $\sim 90 \mathrm{C}$. A $5 \mathrm{~cm}$ diameter sapphire (aluminum oxide) wafer was placed on top of the PLA filaments (Fig. 4). After several minutes, 
as the PLA melted, a small 200 gram weight was used to gently press the sapphire wafer, spreading the melting plastic underneath into a flat sample approximately 0.5 $\mathrm{mm}$ thick. Once fully melted, the Pyrex dish was immediately moved to a benchtop and allowed to cool for exactly $7 \mathrm{~min}$. After cooling, a razor blade was used to carefully lift the PLA sample from the Pyrex dish and remove the sapphire wafer. Scissors sterilized with ethanol were used to cut the sample into $1 \times 3 \mathrm{~cm}$ coupons for biopanning trials, $0.5 \times 0.5 \mathrm{~cm}$ coupons for indirect assays, or $0.75 \times 0.75 \mathrm{~cm}$ coupons for visualization. Sapphire wafer was also used to as an alternate material for comparison after binding trials (described below and depicted in Fig. 3). Sapphire has a smooth, characterized surface and can be cut to size using an etching tool.

\section{Biopanning}

Biopanning rounds consist of several steps: 1) incubating the target material with the naive library culture, 2) washing unbound bacteria from the material surface, 3 ) placing the washed material with bound library members into fresh media, 4) allowing the bound members to amplify (resulting in the Round 1 library), and 5) repeating steps $1-4$ for two total panning rounds. The pB33-dsRed-eCPX3.0 library was prepared for panning as previously described $[6,17,26]$ with modifications depicted in Fig. 3 for the current study. In brief, peptide expression was induced overnight using $0.4 \% \mathrm{~L}$-arabinose after $250 \mathrm{ml}$ overnight stocks diluted 1:50 in $\mathrm{LB}+\mathrm{Cm}$ reached an OD600 0.50-0.60. Induced cells were chilled on ice for $15 \mathrm{~min}$ and transferred to a sterilized crystalizing dish containing four $1 \times 3 \mathrm{~cm}$ PLA samples sterilized with $70 \%$ ethanol. Samples were kept at $4{ }^{\circ} \mathrm{C}$ for $15 \mathrm{~min}$ with shaking to allow bacteria to bind. PLA coupons were removed from the library culture, briefly dipped into sterile phosphate buffered saline (PBS) to remove the bulk of unbound bacteria, and transferred to $20 \mathrm{ml}$ of PBS supplemented with $1 \%$ Tween. Coupons were washed for 30 $\mathrm{min}$ at $330 \mathrm{rpm}$ after incubation with the naive library, and for $1 \mathrm{~h}$ after incubation with the Round 1 library. After washing, bound cells were recovered by placing the PLA coupons into $\mathrm{LB}+\mathrm{Cm}$ media supplemented with $2 \% \mathrm{D}$ Glucose $(\mathrm{LB}+\mathrm{Cm} / \mathrm{Glu})$ and grown at $37{ }^{\circ} \mathrm{C}$ with shaking overnight. Fifty randomly selected colonies from Round 2 were sequenced using the pBAD Reverse universal primer (Genewiz).

\section{Visualization binding assay}

In order to visually compare adhesion across samples after biopanning against PLA, $0.5 \times 0.5 \mathrm{~cm}$ PLA coupons and sapphire pieces were incubated with induced $5 \mathrm{~mL}$ cultures of the naive library, sorting rounds, or negative control (which consists of a library member with an empty eCPX scaffold, i.e. no peptide). Cultures were prepared from freezer stocks grown overnight in $\mathrm{LB}+\mathrm{Cm}$. Fresh $\mathrm{LB}$ was inoculated 1:50 with the overnight stocks and brought to an OD of 0.5-0.6, then induced overnight with $0.4 \%$ arabinose as described above. PLA and sapphire pieces were incubated for $10 \mathrm{~min}$ with each culture at $230 \mathrm{rpm}$, then washed for an additional $10 \mathrm{~min}$ in fresh culture tubes containing $4 \mathrm{ml}$ of PBS $1 \%$ Tween at $330 \mathrm{rpm}$ to remove weakly bound cells. A final dip in $4 \mathrm{ml}$ of fresh PBS ensured any unbound bacteria in the wash solution would be completely removed. Material samples were placed on microscope slides and visualized using an epifluorescent microscope (Nikon Eclipse TE2000-E confocal) at 40X and a red fluorescent filter (AT-TRITC/CY3). Samples were prepared independently in triplicate and ten images were captured from each sample and analyzed for the number of red fluorescent pixels using ImageJ [27]. Student's T-tests were used to statistically verify differences in fluorescent pixel density.

\section{Indirect binding assay}

Indirect binding assays were performed to quantify the number of cells bound to PLA and sapphire pieces as previously described [6]. In brief, incubation of sample materials with libraries and the negative control was the same as described above, however, after washing, the material samples were placed in $6 \mathrm{ml}$ of fresh $\mathrm{LB}+\mathrm{Cm} / \mathrm{Glu}$ and grown for $1 \mathrm{~h}$ at $37^{\circ} \mathrm{C}$ with shaking. Serial dilutions of amplified cells from each sample were plated on $\mathrm{LB}+\mathrm{Cm}$ agar plates. Samples were prepared independently in triplicate and the averages and standard deviations determined. Student's T-tests were used to statistically verify differences in relative binding results.

\section{Next generation sequencing}

Three library culture samples were prepared for Illumina next generation sequencing: the naive library and libraries recovered after each of two rounds of biopanning. Plasmids were extracted from overnight cultures using a ZymoPure plasmid maxiprep kit (Zymo Research) and prepared for sequencing according to the Illumina $16 \mathrm{~S}$ amplicon protocol using custom forward (AGTTCTGGCTTTCACCGCAG) and reverse (CCGTAGTACTGGTTTTTGTTGTAGTC) primers corresponding to eCPX scaffold regions adjacent to the peptide insert and run on a NextSeq500. Primers also included standard Illumina platform adapter overhangs and multiplex barcodes (not shown).

Illumina fastq files were analyzed using modified Matlab scripts based on the format presented in Matochko et al. (2012)[12]. Sequences were parsed into categories based on: 1) valid sequences: inserts that had both correct flanking sequences, were in the correct reading frame, contained no stop codons, and had all bases above the quality threshold (sequences with an Illumina quality 
score above Q20, i.e. bases that have a $99 \%$ or better chance of having been correctly assigned), 2) inserts containing nucleotides with incorrect reading frames, which resulted in a nonfunctional eCPX, 3) inserts in the correct reading frame that contained stop codons, and 4) empty eCPX scaffolds (scaffolds that did not contain any insert; Fig. 5). Valid sequences were then sorted to discover unique sequences. Additional sequence analysis was performed with in-house scripts, and the pLogo probability logo generator [18] was used to study sequence motifs.

\section{Supplementary information}

Supplementary information accompanies this paper at https://doi.org/10.1186/s12896-019-0577-8.

Additional file 1: Figure S1 Amino acids represented within unique sequences. Amino acid representation of all unique sequences within each library $\mathbf{a}$ excluding and $\mathbf{b}$ including the effects of frequency of sequence occurrence for Naive (blue), Round 1 (orange) and Round 2 (grey).

Figure S2 Unique 15-mer sequence motifs from all libraries. Visualization of unique 15-mer sequence motifs calculated against background probabilities for E. coli K12 in a Naive b Round 1 and $\mathbf{c}$ Round 2 libraries, as generated by the pLogo software (http://plogo.uconn.edu) [18]. Figure S3 Unique 15-mer sequence motifs from the Round 2 library and subsets of the Round 2 library. Visualization of unique 15-mer sequence motifs calculated against background probabilities for E. coli K12 in the Round 2 library: a all 15-mers b 40 most frequently occurring 15-mers and c. 10 most frequently occurring 15-mers, as generated by the pLogo software (http://plogo.uconn.edu) [18]. Figure S4 Library growth curves. Growth curves over $6 \mathrm{~h}$ for the naive library, both sorting rounds, and the negative control after a 1:100 dilution from overnight cultures in fresh media. Table S1 Raw count of total and unique sequences for all sequence categories of each library. Table S2 Raw count and decimal of total sequences in library containing $0-4$ cysteines, round by round.

\section{Abbreviations}

NC: Negative control; NGS: Next generation sequencing; NL: Naive library; PLA: Polylactic acid; R1: Round 1; R2: Round 2

\section{Acknowledgements}

We thank Jeffrey Rice for technical discussions and advice.

\section{Authors' contributions}

SDS led experimental design, collected data, performed analyses, and led manuscript preparation. DAS aided in experimental design, data collection, analysis, manuscript preparation, and submission. BLA conceived of the DsRed panning library and aided in experimental design and manuscript preparation. MAH generated the panning library. RLR collected all next generation sequencing data. MMH contributed to data analysis and manuscript preparation. DNS contributed to experimental design, manuscript preparation, and supervised the work. The final manuscript was read and accepted by all co-authors.

\section{Funding}

This work was funded entirely by the U.S. Army Combat Capabilities Development Command Army Research Laboratory (CCDC ARL), in part through contracts with other entities for personnel: the U.S. Army Research Laboratory Postdoctoral Fellowship Program administered by the Oak Ridge Associated Universities and General Technical Services, LLC. As such, all authors were Federal Government employees or contractors when this research was completed. The study design, the collection, analysis, and interpretation of data, as well as the writing of the manuscript, were performed with CCDC ARL funds, facilities, and research personnel. The views expressed here are those of the authors and do not represent the opinions of the U.S. Army, the U.S. Department of Defense, or the U.S. Government.

\section{Availability of data and materials}

The NGS datasets and materials supporting the conclusions of this article are available from the corresponding author upon reasonable request.

\section{Ethics approval and consent to participate}

Not applicable.

\section{Consent for publication}

Not applicable.

\section{Competing interests}

The authors declare that they have no competing interests.

\section{Author details}

${ }^{1}$ Biotechnology Branch, CCDC US Army Research Laboratory, 2800 Powder Mill Rd, Adelphi, MD, 20783 USA. ${ }^{2}$ Department of Biological Sciences, University of Maryland Baltimore County, 1000 Hilltop Circle, Baltimore, MD, 21250 USA. ${ }^{3}$ General Technical Services, Suite 301, 1451 Route 34 South, Wall Township, NJ, 07727 USA.

Received: 1 July 2019 Accepted: 12 November 2019

Published online: 21 December 2019

\section{References}

1. Ibsen KN, Daugherty PS. Prediction of antibody structural epitopes via random peptide library screening and next generation sequencing. J Immunol Methods. 2017;451:28-36.

2. Molek P, Strukelj B, Bratkovic T. Peptide phage display as a tool for drug discovery: Targeting membrane receptors. Molecules. 2011;16(1):. https:// doi.org/10.3390/molecules16010857.

3. Martins IM, Reis RL, Azevedo HS. Phage display technology in biomaterials engineering: Progress and opportunities for applications in regenerative medicine. ACS Chem Biol. 2016;11(11):2962-80.

4. Ploss M, Facey SJ, Bruhn C, Zemel L, Hofmann K, Stark RW, Albert B, Hauer B. Selection of peptides binding to metallic borides by screening m13 phage display libraries. BMC Biotechnol. 2014;14(1):12.

5. Sarkes DA, Hurley MM, Stratis-Cullum DN. Unraveling the roots of selectivity of peptide affinity reagents for structurally similar ribosomal inactivating protein derivatives. Molecules. 2016;21(11):. https://doi.org/ 10.3390/molecules21111504

6. Adams BL, Finch AS, Hurley MM, Sarkes DA, Stratis-Cullum DN. Biomaterials: Genetically engineered peptides for inorganics: Study of an unconstrained bacterial display technology and bulk aluminum alloy (adv. mater. 33/2013). Adv Mater. 2013;25(33):4530.

7. Bessette PH, Rice JJ, Daugherty PS. Rapid isolation of high-affinity protein binding peptides using bacterial display. Protein Eng Des Sel. 2004;17(10) 731-9.

8. Kenrick SA, Daugherty PS. Bacterial display enables efficient and quantitative peptide affinity maturation. Protein Eng Des Sel. 2010;23(1):9-17.

9. Kogot JM, Pennington JM, Sarkes DA, Kingery DA, Pellegrino PM, Stratis-Cullum DN. Screening and characterization of anti-seb peptides using a bacterial display library and microfluidic magnetic sorting. J Mol Recogn. 2014;27(12):739-45.

10. Smith GP, Petrenko VA. Phage display. Chem Rev. 1997;97(2):391-410.

11. Matochko W, Derda R. In: Derda R, editor. Next-Generation Sequencing of Phage-Displayed Peptide Libraries. Methods in Molecular Biology, vol. 1248. Chap. 17: Springer; 2015, pp. 249-66. https://doi.org/10.1007/9781-4939-2020-4_17.

12. Matochko WL, Chu K, Jin B, Lee SW, Whitesides GM, Derda R. Deep sequencing analysis of phage libraries using illumina platform. Methods. 2012:58(1):47-55

13. 't Hoen PAC, Jirka SMG, ten Broeke BR, Schultes EA, Aguilera B, Pang KH, Heemskerk H, Aartsma-Rus A, van Ommen GJ, den Dunnen JT. Phage display screening without repetitious selection rounds. Anal Biochem. 2012;421(2):622-31

14. Daugherty PS. Protein engineering with bacterial display. Curr Opin Struct Biol. 2007;17(4):474-80.

15. Rice JJ, Schohn A, Bessette PH, Boulware KT, Daugherty PS. Bacterial display using circularly permuted outer membrane protein ompx yields high affinity peptide ligands. Protein Sci Publ Protein Soc. 2006;15(4):825-36. 
16. Rice JJ, Daugherty PS. Directed evolution of a biterminal bacterial display scaffold enhances the display of diverse peptides. Protein Eng Des Sel. 2008;21(7):435-42.

17. Sarkes DA, Jahnke JP, Stratis-Cullum DN. Semi-automated biopanning of bacterial display libraries for peptide affinity reagent discovery and analysis of resulting isolates. JoVE (J Visualized Exp). 2017;130:56061. https://doi.org/10.3791/56061.

18. O'Shea JP, Chou MF, Quader SA, Ryan JK, Church GM, Schwartz D. plogo: a probabilistic approach to visualizing sequence motifs. Nat Methods. 2013;10(12):1211.

19. Bosma T, Rink R, Moosmeier MA, Moll GN. Genetically encoded libraries of constrained peptides. ChemBioChem. 2019;20(14):1754-8.

20. Ryvkin A, Ashkenazy H, Smelyanski L, Kaplan G, Penn O, Weiss-Ottolenghi Y, Privman E, Ngam PB, Woodward JE, May GD, Bell C, Pupko T, Gershoni JM. Deep panning: Steps towards probing the igome. PLOS ONE. 2012;7(8):41469.

21. Kilgore HR, Raines RT. $n \rightarrow \pi^{*}$ interactions modulate the properties of cysteine residues and disulfide bonds in proteins. J Am Chem Soc. 2018;140(50):17606-11.

22. Adams BL, Hurley MM, Jahnke JP, Stratis-Cullum DN. Functional and selective bacterial interfaces using cross-scaffold gold binding peptides. JOM. 2015;67(11):2483-93.

23. Sarkes DA, Hurley MM, Coppock MB, Farrell ME, Pellegrino PM, Stratis-Cullum DN. Rapid discovery of peptide capture candidates with demonstrated specificity for structurally similar toxins. In: Proc. SPIE 9863, Smart Biomedical and Physiological Sensor Technology XIII, 986305. International Society for Optics and Photonics; 2016. p. 1-10. https://doi. org/10.1117/12.2224799.

24. Erickson MG, Moon DL, Yue DT. Dsred as a potential fret partner with cfp and gfp. Biophys J. 2003;85(1):599-611.

25. Casadaban MJ, Cohen SN. Analysis of gene control signals by dna fusion and cloning in escherichia coli. J Mol Biol. 1980;138(2):179-207.

26. Kogot JM, Zhang Y, Moore SJ, Pagano P, Stratis-Cullum DN, Chang-Yen D, Turewicz M, Pellegrino PM, de Fusco A, Soh HT, Stagliano NE. Screening of peptide libraries against protective antigen of bacillus anthracis in a disposable microfluidic cartridge. PLoS ONE. 2011;6(11):26925.

27. Schneider CA, Rasband WS, Eliceiri KW. Nih image to imagej: 25 years of image analysis. Nat Methods. 2012;9(7):671-5.

\section{Publisher's Note}

Springer Nature remains neutral with regard to jurisdictional claims in published maps and institutional affiliations.

\section{Ready to submit your research? Choose BMC and benefit from:}

- fast, convenient online submission

- thorough peer review by experienced researchers in your field

- rapid publication on acceptance

- support for research data, including large and complex data types

- gold Open Access which fosters wider collaboration and increased citations

- maximum visibility for your research: over $100 \mathrm{M}$ website views per year

At $B M C$, research is always in progress.

Learn more biomedcentral.com/submissions 